\title{
Skin capillary circulation severely impaired in toes of patients with IDDM, with and without late diabetic complications
}

\author{
G. Jörneskog ${ }^{1}$, K. Brismar ${ }^{2}$, B. Fagrell ${ }^{1}$ \\ ${ }^{1}$ Department of Internal Medicine, Karolinska Institute, Karolinska Hospital, Stockholm, Sweden \\ ${ }^{2}$ Department of Endocrinology and Diabetology, Karolinska Hospital, Stockholm, Sweden
}

\begin{abstract}
Summary We have recently shown that the skin microcirculation of toes is significantly impaired in patients with diabetes and peripheral vascular disease, and this may be one major reason why these patients are highly susceptible to developing skin ulcers. The aim of the present study was to investigate whether the skin microcirculation is impaired also in diabetic patients free from macroangiopathy. One foot in each of 20 patients with insulin-dependent diabetes was investigated: 10 patients with and 10 patients without late complications. All patients had normal arterial circulation of their lower extremities. Two groups of age- and sex-matched healthy subjects served as controls. The capillary blood cell velocity in the nailfold of the great toe was investigated by computerised videophotometric capillaroscopy, and the total microcirculation within the same area evaluated by laser Doppler fluxmetry. The capillary blood cell velocity and the total skin microcirculation were studied during rest, and during postocclusive reactive hyperaemia. The total microcirculation was simi-
\end{abstract}

lar in patients and control subjects, whereas the capillary circulation was markedly reduced $(p<0.01)$ in the patients. The ratio between the capillary and total microcirculation was significantly decreased $(p<0.05-0.01)$ in the patients as compared to the control subjects, indicating a local maldistribution of blood in the skin microcirculation of the diabetic patients. The results of the present study show that in spite of a normal total skin microcirculation in the toes of insulin-dependent diabetic patients, both with and without late complications, the nutritional capillary circulation is severely impaired. These findings indicate that a chronic ischaemia is present in the skin capillaries of diabetic feet, and is related to the diabetic disease per se and not to late diabetic complications, and may be a cause for these complications. [Diabetologia (1995) 38: 474-480]

Key words Diabetes mellitus, skin microcirculation, capillary blood cell velocity, laser Doppler fluxmetry.
Several processes characteristic of the diabetic disease work together resulting in a condition called 'the diabetic foot'. This constellation consists of macroangiopathy, peripheral neuropathy, haemorheological and metabolic disturbances, which are com-

Received: 4 October 1994

Corresponding author: Dr. G. Jörneskog, Department of Internal Medicine, Karolinska Hospital, S-17176 Stockholm, Sweden

Abbreviations: PVD, Peripheral vascular disease; IDDM, insulin-dependent diabetes mellitus; CBV, capillary blood cell velocity; LDF, laser Doppler fluxmetry. bined to a varying extent and can result in severe nutritional disturbances leading to, e. g. bone degeneration and chronic ulcers in the foot [1]. A sufficient blood supply to the affected tissue is one essential factor for the healing process of ulcers. Normally, the main part of the skin blood flow is used for thermoregulation of the body, especially in the digits, and less than $10 \%$ of the total skin blood flow passes through the nutritional skin capillaries [2-4]. However, a severely reduced blood flow through the nutritional skin capillaries has been found in patients with peripheral vascular disease (PVD), despite the fact that the total blood flow could be normal or even increased $[5,6]$. We have recently shown that this mal- 
Table 1. Clinical details of the diabetic patients with (+ Compl) and without (No compl) complications and their respective control subjects

\begin{tabular}{lcccc}
\hline & Diabetes + no compl & Control group 1 & Diabetes + compl & Control group 2 \\
\hline Sex (female: male) & $5: 5$ & $5: 5$ & $2: 8$ & $2: 8$ \\
Age (years) & $29.9 \pm 7.0$ & $30.2 \pm 7.9$ & $46.1 \pm 9.8$ & $47.3 \pm 9.2$ \\
BMI (kg/m $\left.{ }^{2}\right)$ & $22.8 \pm 2.7$ & $22.5 \pm 2.9$ & $23.5 \pm 3.1$ & $22.7 \pm 2.3$ \\
Smokers $(n)$ & 5 & 3 & 6 & 3 \\
Duration of diabetes (years) & $4.8 \pm 2.9$ & & 6 & 7 \\
Non-healing foot ulcers $(n)$ & 0 & & 3 \\
Retinopathy $(n)$ & 0 & & 3.6 & \\
Microalbuminuria $(n)$ & 0 & & & \\
\hline
\end{tabular}

Results expressed as number $(n)$, or mean $\pm \mathrm{SD}$

Table 2. Peripheral blood pressure measurements in the diabetic patients and their respective control subjects

\begin{tabular}{lccrr}
\hline & Diabetes + no compl & Control group 1 & Diabetes + compl & Control group 2 \\
\hline Arm systolic blood pressure $(\mathrm{mmHg})$ & $123 \pm 11.4$ & $121 \pm 9.7$ & $133 \pm 15.1$ & $128 \pm 16.9$ \\
Arm diastolic blood pressure $(\mathrm{mmHg})$ & $77 \pm 4.8$ & $76 \pm 5.5$ & $81 \pm 10.5$ & $80 \pm 11.5$ \\
Ankle blood pressure $(\mathrm{mmHg})$ & $117 \pm 13.1$ & $122 \pm 7.5$ & $141 \pm 13.6$ & $140 \pm 23.1$ \\
Toe blood pressure (mmHg) & $113 \pm 13.5$ & $115 \pm 17.7$ & $123 \pm 19.6$ & $122 \pm 19.0$ \\
Toe/arm blood pressure index & $0.90 \pm 0.12$ & $0.95 \pm 0.12$ & $0.94 \pm 0.23$ & $0.96 \pm 0.13$ \\
\hline
\end{tabular}

Results expressed as mean $\pm \mathrm{SD}$

Table 3. Cardiovascular autonomic function tests, and vibration perception threshold of the great toe in the diabetic patients with (+ Compl) and without (No compl) complications

\begin{tabular}{|c|c|c|}
\hline & $\begin{array}{l}\text { Diabetes + } \\
\text { no compl }\end{array}$ & $\begin{array}{l}\text { Diabetes + } \\
\text { compl }\end{array}$ \\
\hline $\begin{array}{l}\text { Heart rate variation during } \mathrm{d} \\
\text { Exspiration/inspiration ratio } \\
\text { (reference value }^{a} \text { ) }\end{array}$ & $\begin{array}{l}\text { p breathing } \\
1.39 \pm 0.12 \\
(>1.26 \pm 0.04)\end{array}$ & $\begin{array}{c}1.08 \pm 0.05 \\
(>1.18 \pm 0.05)\end{array}$ \\
\hline $\begin{array}{l}\text { Heart rate response to tilt } \\
\text { Acceleration index } \\
(\text { reference value })^{a}\end{array}$ & $\begin{array}{c}26.2 \pm 8.8 \\
(>14.2 \pm 2.3)\end{array}$ & $\begin{array}{c}8.6 \pm 6.9 \\
(>9.2 \pm 2.8)\end{array}$ \\
\hline $\begin{array}{l}\text { Brake index } \\
\left.\text { (reference value }^{a}\right)\end{array}$ & $\begin{array}{c}24.3 \pm 9.9 \\
(>11.7 \pm 2.5)\end{array}$ & $\begin{array}{c}5.4 \pm 5.6 \\
(>6.1 \pm 3.1)\end{array}$ \\
\hline $\begin{array}{l}\text { Vibration perception } \\
\text { threshold (V) } \\
\left.\text { (reference value }^{b}\right)\end{array}$ & $\begin{array}{c}9.5 \pm 2.1 \\
(<10.7 \pm 1.8)\end{array}$ & $\begin{array}{c}36.7 \pm 9.5 \\
(<17.0 \pm 4.6)\end{array}$ \\
\hline
\end{tabular}

Results expressed as mean $\pm \mathrm{SD}$.

${ }^{a}$ For age-related normal values see reference 13 .

${ }^{\mathrm{b}}$ For age-related normal values see reference 43

distribution of skin blood flow is even more pronounced in diabetic patients with PVD, and that the postocclusive reactive hyperaemia response is severely impaired [7]. A steal phenomenon, where the blood bypasses the nutritional skin capillaries [8], may be one of the most important factors for the development of tissue ischaemia in diabetic feet, but whether this maldistribution of blood is present also in diabetic patients without PVD is not fully known.

The aim of the present study was to investigate the total and the capillary skin microcirculation in toes of insulin-dependent diabetic patients clinically free from macroangiopathy in the lower extremities, in or- der to investigate whether also these diabetic patients, in spite of a normal macrocirculation, have a maldistribution of blood in the skin microcirculation, which could explain their increased rish for developing non-healing foot ulcers. The total skin microcirculation, mainly the circulation in the subpapillary vascular bed [9], was evaluated by laser Doppler fluxmetry in an area known to be rich in arteriovenous anastomoses, i. e. the toe nailfold [2]. Simultaneously, the capillary circulation was measured in the same area by a computerised technique of clinical capillaroscopy [3]. This combination makes it possible to investigate the distribution of blood flow between the non-nutritional, subpapillary microvascular compartments of the skin, and the nutritional skin capillaries.

\section{Subjects and methods}

Subjects. Two groups of IDDM patients, one with and one without late complications, were investigated. Clinical data are presented in Table 1. All patients were treated with intermittent doses of insulin (three to four times daily). The patients with foot ulcers were also on antibiotics, but otherwise no medication was given. All patients were clinically free from PVD, as evaluated by segmental blood pressure measurements $[10,11]$ (Table 2). The autonomic nerve function was evaluated by heart rate variation during deep breathing and the heart rate reaction to tilt $[12,13]$ (Table 3). Peripheral neuropathy was assessed by examining the ankle reflexes, and by measuring vibration perception thresholds of the great toe by biothesiometry [14, 15] (Bio-Medical Instrument Company, Newbury, Ohio, USA) (Table 3). Urine was collected in overnight samples for determination of microalbuminuria [16], and the eyes were examined by an ophthalmologist with ophthalmoscopy and fundus photography. In the patients without 
late complications we also measured the nerve conduction velocity in the posterior tibial, the sural, and the median nerves.

Diabetic patients without late complications (Table 1). This group comprised one foot of each of 10 patients ( 5 males) with a mean age of 29.9 (range $21-35$ ) years and a mean diabetes duration of 4.8 (range 2-10) years. All patients had normal cardiovascular autonomic nerve functions, elicitable ankle jerks, and normal vibration perception tests of the great toe (Table 3 ). Four patients had a slightly prolonged conduction velocity in some of the investigated nerves, most probably indicating a very early stage of neuropathy. None had microalbuminuria or retinopathy.

Diabetic patients with late complications (Table 1). This group consisted of one foot of each of 10 patients ( 8 males) with a mean age of 46.1 (range 30-54) years and a mean diabetes duration of 17.7 (range 6-33) years. All patients had evidence of autonomic neuropathy, and the vibration perception test of the great toe was abnormal (Table 3 ). None had elicitable ankle jerks, and six patients had non-healing foot ulcers for at least 2 (range 2-12) months. The ulcers were located under the metatarsal heads, and were classified as neuropathic ulcers. Three patients had microalbuminuria in overnight urine samples (> $20 \mathrm{mg} / 12 \mathrm{~h}$ ), two patients proliferative retinopathy, and five patients background retinopathy.

Control subjects (Table 1). Two control groups were investigated. Control group 1 consisted of 10 feet of 10 age- and sexmatched healthy subjects to the patients without complications, and control group 2 comprised 10 feet of 10 age- and sex-matched healthy subjects to the patients with late diabetic complications. None of the healthy subjects had a family history of diabetes.

Methods. The skin microcirculation in the nailfold of the great toe was investigated by computerised videophotometric capillaroscopy $[3,17,18]$ and laser Doppler fluxmetry $[9,19]$. All subjects were acclimatized for at least $30 \mathrm{~min}$ before the investigations started, and the room temperature was kept between $22-24^{\circ} \mathrm{C}$. All participants were asked to refrain from smoking and coffee drinking the day of the study. The subjects were investigated in the supine position with the knees slightly flexed and the legs comfortably resting in a special holder to avoid involuntary movements of the foot. A miniature cuff $(20 \mathrm{~mm}$ wide) was applied at the proximal phalanx of the great toe so that arterial occlusions could be performed. The skin temperature of the investigated toe nailfold was continuously recorded with an electronic thermistor (Exacon, Copenhagen, Denmark).

Videophotometric capillaroscopy. Nailfold capillaries of the great toe were visualized on a TV-monitor by a Leitz Laborlux microscope (Leica (Leitz), Wetzlar, Germany) on which a CCD video camera (ICD-44 DC, Ikegami, Tokyo, Japan) was mounted. The image was stored on videotape for subsequent analysis. The capillary blood cell velocity (CBV) was determined by a computerised, videophotometric, cross-correlation technique [3, 17, 18] (Capiflow AB, Stockholm, Sweden). The CBV was measured in a suitable capillary with good contrast and visible signals. This has been shown to be relevant for studying skin microvascular reactivity [20]. The following variables were determined; resting CBV $(\mathrm{mm} / \mathrm{s})$; peak CBV $(\mathrm{mm} /$ s) and time to peak CBV (s) following release of a 1-min arterial occlusion at the proximal phalanx of the great toe with a cuff pressure of $200 \mathrm{~mm} \mathrm{Hg}$; per cent increase of resting CBV (CBV \%) during postocclusive reactive hyperaemia.
The reproducibility of the capillaroscopic technique used was tested in the present study. Duplicate measurements of post-occlusive reactive hyperaemia with 2-3 min separation were performed in 30 subjects. There was a strong linear association for peak CBV $(r=0.98, p<0.001)$, and a significant correlation for time to peak CBV between the two measurements $(r=0.92, p<0.001)$. No systematic difference was observed between the two measurements (paired $t$-test), either for peak $\operatorname{CBV}(p=0.501)$, or for time to peak CBV $(p=0.814)$.

Laser Doppler fluxmetry. The total skin microcirculation was measured by laser Doppler fluxmetry (LDF) (Periflux, Pf 1d, Perimed, Stockholm, Sweden) simultaneously with videophotometric capillaroscopy [19]. The laser Doppler output signal, which to more than $90 \%$ is generated by flow in subpapillary vessels [9], was continuously recorded on a pen recorder and full scale deflection was 10 Volt. A bandwidth of $4 \mathrm{khz}$ and a gain of 10 times were used. The laser Doppler probe was placed within the skin area immediately adjacent to the microscopic field of view, and the following variables were measured: resting LDF (V); peak LDF (V), time to peak LDF (s), and per cent increase of resting LDF (LDF \%) after a one minute arterial occlusion at the toe base. The remaining flux signal during the arterial occlusion was considered to be the biological zero value (V), which was subtracted from the total laser Doppler signal $[9,21]$.

The reproducibility was also tested in the present study for the LDF method. Duplicated measurements of postocclusive reactive hyperaemia with 2-3 min intervals were performed in 26 subjects. There was a strong linear association for peak LDF $(r=0.98, p<0.001)$, and no systematic difference between the two measurements was observed $(p=0.464)$. The time to peak LDF showed also a significant correlation between the two measurements $(r=0.92, p<0.001)$, and no systematic difference was noticed $(p=0.645)$ between the two measurements with paired $t$-test.

Blood tests. Venous blood was taken for determination of haemoglobin, haematocrit, blood glucose, glycated haemoglobin $\left(\mathrm{HbA}_{1 \mathrm{c}}\right)$, plasma fibrinogen, serum cholesterol and serum triglyceride. Blood glucose was measured by the glucosoxidase/ peroxidase method using dry chemistry (Kodak Ektachem Clin Chem, Rochester, Mn., USA); $\mathrm{HbA}_{1 \mathrm{c}}$ by the ELISAmethod using monoclonal antibodies (Dakopatts, DAKO Diagnostics Ltd. Cambridge, UK); plasma fibrinogen by a fibrinpolymerization rate assay (Fibri-Prest Automate, Stago, Asnielres, France); serum cholesterol and serum triglyceride were measured by dry chemistry (Kodak Ektachem Clin Chem); microalbuminuria by a nephelometric assay (Array, Beckman Instruments, Brea, Calif., USA).

\section{Statistical analysis}

Data are given as mean \pm SD. The Mann-Whitney $U$ test was used to test differences between the groups. A value of $p<0.05$ was considered statistically significant. The study was approved by the ethics committee of the Karolinska Hospital.

\section{Results}

Skin temperature (Table 4). The skin temperature was similar in all four groups, and did not change during the investigation. 
Table 4. Microcirculatory data in the diabetic patients and their respective control subjects

\begin{tabular}{llccc}
\hline & Diabetes + no compl & Control group 1 & Diabetes + compl & Control group 2 \\
\hline Skin temperature ( $\left.{ }^{\circ} \mathrm{C}\right)$ & $27.3 \pm 2.0$ & $28.7 \pm 1.4$ & $29.0 \pm 2.3$ & $28.9 \pm 2.8$ \\
Resting CBV (mm/s) & $0.09 \pm 0.05^{\mathrm{b}}$ & $0.33 \pm 0.24$ & $0.17 \pm 0.12$ & $0.35 \pm 0.42$ \\
Peak CBV (mm/s) & $0.18 \pm 0.14^{\mathrm{b}}$ & $0.61 \pm 0.39$ & $0.14 \pm 0.07^{\mathrm{c}}$ & $0.63 \pm 0.40$ \\
Time to peak CBV (s) & $19.7 \pm 9.1$ & $14.5 \pm 6.3$ & $19.9 \pm 9.7$ & $14.5 \pm 5.7$ \\
CBV\% & $102 \pm 157$ & $93 \pm 52$ & $31 \pm 112^{\mathrm{a}}$ & $153 \pm 122$ \\
Resting LDF (V) & $1.5 \pm 1.3$ & $1.7 \pm 1.6$ & $2.8 \pm 2.5$ & $1.2 \pm 0.8$ \\
Peak LDF (V) & $2.7 \pm 2.0$ & $2.8 \pm 1.7$ & $3.8 \pm 2.8$ & $3.2 \pm 2.2$ \\
Time to peak LDF (s) & $9.1 \pm 3.1$ & $8.8 \pm 3.0$ & $9.7 \pm 6.4$ & $8.1 \pm 4.7$ \\
LDF\% & $171 \pm 194$ & $127 \pm 109$ & $85 \pm 96$ & $175 \pm 125$ \\
Biological zero (V) & $0.10 \pm 0.12$ & $0.21 \pm 0.11$ & $0.12 \pm 0.08$ & $0.19 \pm 0.12$ \\
Resting CBV/resting LDF & $0.12 \pm 0.11^{\mathrm{a}}$ & $0.35 \pm 0.30$ & $0.11 \pm 0.10^{\mathrm{a}}$ & $0.37 \pm 0.43$ \\
Peak CBV/peak LDF & $0.13 \pm 0.19^{\mathrm{a}}$ & $0.32 \pm 0.28$ & $0.07 \pm 0.09^{\mathrm{b}}$ & $0.24 \pm 0.14$ \\
\hline
\end{tabular}

Values are given as mean $\pm \mathrm{SD}$.

${ }^{\mathrm{a}} p<0.05,{ }^{\mathrm{b}} p<0.01,{ }^{\mathrm{c}} p<0.001$ as compared to healthy control subjects

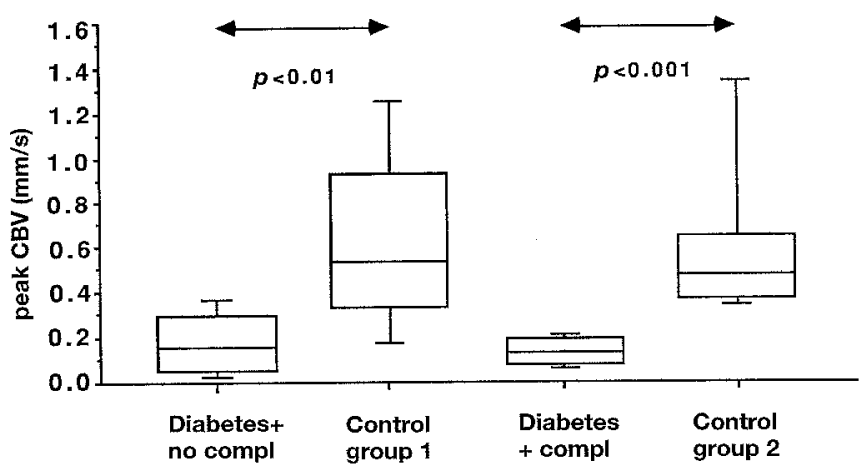

Fig. 1. Peak capillary blood cell velocity (CBV) in diabetic patients with $(+\mathrm{Compl})$ and without (No compl) complications and their respective control subjects. Box-plot values of peak CBV showing median values and the 10 th, 25 th, 75 th and 90 th percentiles

Total skin microcirculation (Table 4). Resting LDF, peak LDF; time to peak LDF and LDF \% were similar in both patients and control subjects although there was a tendency for higher LDF-values in the patients with complications. The biological zero value was small and similar in all groups. No differences were found between patients with and without foot ulcerations regarding the LDF variables.

Capillary circulation (Table 4). Resting CBV was lower $(p<0.01)$ in the diabetic patients without complications, as compared to the control subjects, while resting $\mathrm{CBV}$ in the diabetic patients with complications did not differ from the control subjects. Peak CBV was markedly decreased in both patient groups $(p<0.01)$, as compared to the control subjects (Fig.1), while time to peak CBV was not significantly different from the control subjects. $\mathrm{CBV} \%$ was significantly lower in the diabetic patients with complications $(p<0.05)$ as compared to the control subjects, while it was similar to control subjects in the diabetic patients without complications.

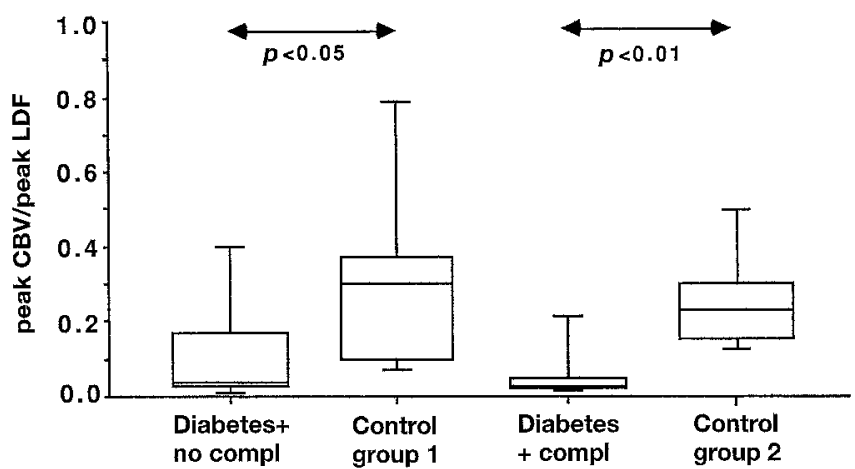

Fig. 2. Ratio between peak blood flow in nutritional (CBV) and non-nutritional (LDF) microvascular compartments in diabetic patients with (+ Compl) and without (No compl) complications and their respective control subjects. Boxplots, see Figure 1

The ratio between CBV and LDF, representing the distribution of blood between nutritional and nonnutritional microvascular compartments, was significantly lower $(p<0.05)$ in both patient groups as compared to the control subjects, both during rest and reactive hyperaemia (Fig.2).

No differences were found in the CBV variables between patients with and without foot ulcerations.

Blood tests (Table 5). Blood glucose was similar in both patient groups and significantly higher $(p<0.001)$ as compared to control subjects. $\mathrm{HbA}_{1 \mathrm{c}}$ was equally elevated in the patient groups. No significant correlations were found between the microvascular variables and the values of blood glucose and $\mathrm{HbA}_{1 \mathrm{c}}$ respectively. Haemoglobin, haematocrit, plasma fibrinogen, serum cholesterol and serum triglyceride were similar in patients and control subjects. 
Table 5. Blood tests in the diabetic patients and their respective control subjects

\begin{tabular}{lcccc}
\hline & Diabetes + no compl & Control group 1 & Diabetes + compl & Control group 2 \\
\hline Haemoglobin $(120-150 \mathrm{~g} / \mathrm{l})$ & $144 \pm 10$ & $139 \pm 10$ & $138 \pm 17$ & $145 \pm 10$ \\
Haematocrit $(37-43 \%)$ & $42 \pm 3.0$ & $41 \pm 2.9$ & $40 \pm 4.8$ & $42+2.7$ \\
Blood glucose $(3-6 \mathrm{mmol} / \mathrm{l})$ & $11.3 \pm 6.5^{\mathrm{a}}$ & $4.5 \pm 0.4$ & $9.7 \pm 1.3^{\mathrm{a}}$ & $4.7 \pm 0.6$ \\
$\mathrm{HbA}_{1 \mathrm{c}}(2.5-4.0 \%)$ & $6.8 \pm 1.0$ & & $7.6 \pm 1.5$ & \\
Plasma fibrinogen $(2.1-4.2 \mathrm{~g} / \mathrm{l})$ & $3.1 \pm 0.6$ & $2.8 \pm 0.7$ & $3.4 \pm 1.1$ & $2.7 \pm 0.4$ \\
Serum cholesterol $(<5.5 \mathrm{mmol} / \mathrm{l})$ & $4.9 \pm 0.9$ & $4.4 \pm 0.7$ & $5.2 \pm 1.0$ & $5.1 \pm 1.1$ \\
Serum triglycirides $(0.6-2.2 \mathrm{mmol} / \mathrm{l})$ & $1.0 \pm 0.4$ & $1.0 \pm 0.3$ & $0.9 \pm 0.2$ & $1.2 \pm 0.4$ \\
\hline
\end{tabular}

Normal reference values in parentheses. Values are given as mean $\pm \mathrm{SD} .{ }^{a} p<0.001$ as compared to healthy control subjects

\section{Discussion}

A common observation in diabetic patients, particularly those with peripheral neuropathy, is a hot and red foot with easily palpable pulses. Despite this apparently adequate blood supply these patients sometimes develop non-healing foot ulcers. In agreement with these observations several studies have shown that diabetic patients have an increased blood flow in the extremities [22-24], and also an increase of the total skin microcirculation $[25,26]$. Peripheral neuropathy, in combination with repeated mechanical stress, is considered the main reason why chronic foot ulcers develop in skin areas with an increased or normal blood flow, but another contributing factor may be a maldistribution of blood between the nutritional capillaries and the deeper subpapillary vessels. Peripheral sympathetic denervation of precapillary vessels most probably leads to an opening of arteriovenous connections [27], resulting in blood bypassing the nutritional skin capillaries. This hypothesis is strengthened by the finding of an increased venous oxygenation in the diabetic foot [8], but how this maldistribution influences the circulation in the nutritional capillaries is not fully known.

The results of the present study clearly show that a marked maldistribution of blood between the nutritional capillary and subpapillary microvascular compartments actually exists in the skin microcirculation of diabetic feet, with a striking reduction of maximal blood flow in the nutritional skin capillaries. This impairment of capillary blood flow may lead to an impairment of the regional exchange of nutrients and oxygen, which has also been indicated in other investigations [28]. The fact that the reduction in capillary blood flow was more pronounced during the postocclusive reactive hyperaemia than during basal conditions indicates that the capillary ischaemia is more severe during stress situations when the nutritional demand is increased, e.g. during walking and increased pressure from shoes. Flynn et al. [29] have recently investigated the resting $\mathrm{CBV}$ in the toe nailfolds of diabetic patients with neuropathy, and could not find any differences as compared to healthy subjects. By mea- surements of capillary diameter they also estimated the capillary blood flow to be increased in the patients. However, the skin temperature in their diabetic patients was $5^{\circ} \mathrm{C}$ higher than in the control subjects, and as $C B V$ is positively correlated to skin temperature $[4,30], C B V$ in their patients should have been significantly increased compared to the control subjects. As this was not the case, CBV must have been significantly reduced in the diabetic patients. In our study skin temperature was similar in all groups and this is most probably the reason why our results differ from theirs.

One of the most interesting observations in the present study is that the capillary ischaemia in skin of diabetic feet seems to be present already at an early stage of diabetes, as the patients with only 5 years' diabetes duration also showed this maldistribution. Similar findings of impaired microvascular reactivity in forearm skin have been demonstrasted by transcutaneous oxygen tension measurements in children with short-term diabetes [31]. This indicates that the impaired skin capillary circulation is related to the metabolic disturbances and is not a secondary phenomenon to the diabetic complications. A decreased capillary circulation and an increased arterio-venous shunting has been found also in other tissues of the lower extremity in diabetic patients, e.g. the vasa nervorum of the sural nerve [32], and these findings also support the suggestion that microvascular disturbances contribute to the diabetic foot complications. A successive deterioration of the nerve function with neuropathy as the final state could be the consequence of ischaemia also in nutritional capillaries to the nerves $[32,33]$. This is further supported by the finding that the four patients in the present study who had a slightly prolonged nerve conduction velocity also had a severely impaired capillary circulation despite no other evidence of diabetic complications. The extremely high blood flow seen in diabetic patients with osteoarthropathy has been explained by sympathetic denervation of arterioles, leading to an increase in blood flow and rarefacation of bone $[1$, $34,35]$. Our findings of the present study suggest that another contributing factor to the degeneration of bone tissue in these patients may also be an im- 
paired circulation in the nutritional capillaries of the bone tissue.

The exact mechanism behind the maldistribution of blood between nutritional and non-nutritional microvessels is not fully known, but several factors are most probably involved. Long-term hyperglycaemia causes endothelial and metabolic disturbances leading to an elevated blood circulation, capillary hypertension and increased vascular leakage $[24,36,37$, $26,38]$, which may progress to irreversible structural changes [39], such as a thickening of the capillary basement membrane [40]. An increased capillary blood pressure, which has been demonstrated in both the toe and finger nailfolds of diabetic patients $[26,38]$, may be a consequence of an opening of arteriovenous shunt vessels with a transformation of the arteriolar pressure out into the subpapillary venular plexus, resulting in a decreased arteriovenous pressure difference. Since the skin capillaries are rigid tubes $[2,41]$, such a reduced pressure difference will result in a reduced capillary blood flow, leading to hampered tissue nutrition. Haemorheological disturbances, such as a reduced erythrocyte deformability [42], endothelial cellular dysfunction [37], increased blood viscosity and fibrinogen levels $[43,44]$, are additional factors which may further enhance the impaired capillary function.

In conclusion, we have shown that a local microvascular dysfunction with a maldistribution of blood between non-nutritional and nutritional skin vessels is present in the feet of IDDM patients. This maldistribution seems to be more related to the diabetic disease per se than to diabetic complications. As the skin of the toes and feet is exposed to great stress, the disturbed circulation in the nutritional capillaries may generate a regional ischaemia contributing to the syndrome known as 'the diabetic foot' [1]. If the described capillary dysfunction is a primary cause for the diabetic foot complications new therapeutic modalities may improve the prognosis for these patients.

Acknowledgements. This work was supported by grants from Swedish Medical Research Council (6835 and 8797), Swedish Society of Medicine, Swedish Heart Lung Foundation, Swedish Diabetes Association, Swedish Hoechst Company, 'Förenade Liv' Mutual Group Life Insurance Company, Karolinska Institute and King Gustaf V's and Queen Victoria's Foundation. The authors wish to thank Ms A.-C. Salomonsson for excellent technical assistance in this study.

\section{References}

1. Edmonds ME, Watkins PJ (1992) The diabetic foot. In: Alberti KGMM, DeFronzo RA, Keen H, Zimmet P (eds) International textbook of diabetes mellitus, Vol 2. Wiley, Chichester, pp 1535-1549

2. Conrad MC (1971) Functional anatomy of the circulation of the lower extremities. Year book Medical Publishers Inc., Chicago
3. Bollinger A, Fagrell B (1990) Clinical capillaroscopy - a guide to its use in clinical research and practice. Hogrefe \& Huber Publishers, Lewiston

4. Östergren J (1984) Studies on skin capillary blood cell velocity by videophotometric capillaroscopy. Thesis. Repro Print AB, Stockholm

5. Fagrell B (1986) Microcirculatory methods for evaluating the effect of vasoactive drugs in clinical practice. Acta Pharmacol Toxicol 59 [Suppl VI]: 103-107

6. Mc Ewan AJ, Ledingham I McA (1971) Blood flow characteristics and tissue nutrition in apparently ischaemic feet. BMJ 3: 220-224

7. Jörneskog G, Brismar K, Fagrell B (1995) Skin capillary circulation is more impaired in toes of diabetic than non-diabetic patients with peripheral vascular disease. Diabet Med 12: 36-41

8. Boulton AJM, Scarpello JHB, Ward JD (1982) Venous oxygenation in the diabetic neuropathic foot: evidence of arteriovenous shunting? Diabetologia 22: 6-8

9. Fagrell B (1994) Problems using laser Doppler on the skin in clinical practice. Part 1. In: Belcaro G, Hoffman U, Bollinger A, Nicolaides A (eds) Laser Doppler. Med-Orion Publ. Co., London, pp 49-54

10. Gundersen J (1972) Segmental measurements of systolic blood pressure in the extremities including the thumb and the great toe. Acta Chir Scand [Suppl] 426

11. Rutherford RB (1979) Combining segmental systolic pressures and plethysmography to diagnose arterial occlusive disease of the legs. Am J Surg 138: 211-218

12. Sundkvist G, Almer L-O, Lilja B (1979) Respiratory influence on heart rate in diabetes mellitus. BMJ 1: 924-925

13. Bergström B, Lilja B, Rosberg K, Sundkvist G (1986) Autonomic nerve function tests. Reference values in healthy subjects. Clinical Physiology 6: 523-528

14. Guy RJC, Clark CA, Malcolm PN, Watkins PJ (1985) Evaluation of thermal and vibration sensation in diabetic neuropathy. Diabetologia 28: 131-137

15. Bloom S, Till S, Sonksen P, Smith S (1984) Use of biothesiometer to measure individual vibration thresholds and their variation in 519 non-diabetic subjects. BMJ 288: 17931795

16. Rowe DJF, Dawnay A, Watts GF (1990) Microalbuminuria in diabetes mellitus: review and recommendations for the measurements of albumin in urine. Ann Clin Biochem 27: 297-302

17. Fagrell B, Eriksson S-E, Malmström S, Sjölund A (1988) Computerized data analysis of capillary blood cell velocity. Int J Microcirc: Clin Exp 7:276

18. Fagrell B, Fronek A, Intaglietta M (1977) A microscope television system for studying flow velocity in human skin capillaries. Am J Physiol 233 (2): H318-H321

19. Tooke JE, Östergren J, Fagrell B (1983) Synchronous assessment of human skin microcirculation by laser Doppler flowmetry and dynamic capillaroscopy. Int J Microcirc: Clin Exp 2: 277-284

20. Richardson D, Schwartz R (1984) Comparison of resting capillary flow dynamics in the finger and toe nailfolds. Microcirculation, Endothelium, and Lymphatics 1: 645-656

21. Caspary L, Creutzig A, Alexander K (1988) Biological zero in laser Doppler fluxmetry. Int J Microcirc: Clin Exp 7: 367-371

22. Scarpello JHB, Martin. TRP, Ward JD (1980) Ultrasound measurements of pulse-wave velocity in the peripheral arteries of diabetic patients. Clin Sci Mol Med 58: 53-57

23. Archer AG, Roberts VC, Watkins PJ (1984) Blood flow patterns in painful diabetic neuropathy. Diabetologia 27: $563-567$ 
24. Gundersen HJG (1974) Peripheral blood flow and metabolic control in juvenile diabetes. Diabetologia 10: 225-231

25. Rayman G, Hassan A, Tooke JE (1986) Blood flow in the skin of the foot related to posture in diabetes. BMJ 292 : 87-89

26. Rayman G, Williams S, Hassan A, Gamble J, Tooke JE (1985) Capillary hypertension and overperfusion in the feet of young diabetics. Diabet Med 2: 304

27. Watkins PJ, Edmonds ME (1983) Sympathetic nerve failure in diabetes. Diabetologia 25: 75-77

28. Ditzel J (1976) Oxygen transport impairment in diabetes. Diabetes 25 [Suppl 2]: 832-838

29. Flynn MD, Edmonds ME, Tooke JE, Watkins PJ (1988) Direct measurement of capillary blood flow in the diabetic neuropathic foot. Diabetologia 31: 652-656

30. Flynn MD, Hassan AAK, Tooke JE (1989) Effect of postural change and thermoregulatory stress on the capillary microcirculation of the human toe. Clin Sci Mol Med 76: $231-236$

31.Kobbah AM (1988) Early vascular and platelet changes in childhood diabetes. Thesis, Uppsala University, Sweden

32. Tesfaye S, Malik R, Ward JD (1994) Vascular factors in diabetic neuropathy. Diabetologia 37: 847-854

33. Newrick PG, Wilson AJ, Jakubowski J, Boulton AJM, Ward JD (1986) Sural nerve oxygen tension in diabetes. BMJ 293: 1053-1054

34. Eymontt MJ, Alavi A, Dalinka MK, Kyle GC (1981) Bone scintigraphy in diabetic osteoarthropathy. Radiology 140: $475-477$

35. McClugage SG, McCuskey RS (1973) Relationship of the microvascular system to bone resorption and growth in situ. Microvasc Res 6: 132-134
36. Jaap AJ, Shore AC, Gartside IB, Gamble J, Tooke JE (1993) Increased microvascular fluid permeability in young type 1 (insulin-dependent) diabetic patients. Diabetologia 36: 648-652

37. Porta M, La Selva M, Molinatti P, Molinatti GM (1987) Endothelial cell function in diabetic microangiopathy. Diabetologia 30: 601-609

38. Sandeman DD, Shore AC, Tooke JE (1992) Relation of skin capillary pressure in patients with insulin-dependent diabetes mellitus to complications and metabolic control. N Engl J Med 327: 760-764

39. Williamsson JR, Chang K, Frangos M et al. (1993) Hyperglycemic pseudohypoxia and diabetic complications. Diabetes 42: 801-813

40. Williamson JR, Kilo C (1977) Current status of capillary basement-membrane disease in diabetes mellitus. Diabetes $26: 65-73$

41. Murphy ME, Johnson PC (1975) Possible contribution of basement membrane to the structural rigidity of blood capillaries. Microvasc Res 9: 242-245

42. McMillan DE, Utterbach NG, La Puma J (1978) Reduced erythrocyte deformability in diabetes. Diabetes 27: 895-901

43. Lowe GDO, Lowe JM, Drummond MM et al. (1980) Blood viscosity in young male diabetics with and without retinopathy. Diabetologia 18: 359-363

44. Ganda OP, Arkin CF (1992) Hyperfibrinogenemia: an important risk factor for vascular complications in diabetes. Diabetes Care 15: 1245-1250 